\title{
Front Matter: Volume 7583
}

, "Front Matter: Volume 7583," Proc. SPIE 7583, High-Power Diode Laser Technology and Applications VIII, 758301 (22 March 2010); doi: $10.1117 / 12.855217$

SPIE. Event: SPIE LASE, 2010, San Francisco, California, United States 


\section{PROCEEDINGS OF SPIE}

\section{High-Power Diode Laser Technology and Applications VIII}

Mark S. Zediker

Editor

25-26 January 2010

San Francisco, California, United States

Sponsored and Published by

SPIE

Volume 7583

Proceedings of SPIE, 0277-786X, v. 7583 
The papers included in this volume were part of the technical conference cited on the cover and title page. Papers were selected and subject to review by the editors and conference program committee. Some conference presentations may not be available for publication. The papers published in these proceedings reflect the work and thoughts of the authors and are published herein as submitted. The publisher is not responsible for the validity of the information or for any outcomes resulting from reliance thereon.

Please use the following format to cite material from this book:

Author(s), "Title of Paper," in High-Power Diode Laser Technology and Applications VIII, edited by Mark S. Zediker, Proceedings of SPIE Vol. 7583 (SPIE, Bellingham, WA, 2010) Article CID Number.

ISSN 0277-786X

ISBN 9780819479792

Published by

SPIE

P.O. Box 10, Bellingham, Washington 98227-0010 USA

Telephone +1 3606763290 (Pacific Time) · Fax +1 3606471445

SPIE.org

Copyright (c) 2010, Society of Photo-Optical Instrumentation Engineers

Copying of material in this book for internal or personal use, or for the internal or personal use of specific clients, beyond the fair use provisions granted by the U.S. Copyright Law is authorized by SPIE subject to payment of copying fees. The Transactional Reporting Service base fee for this volume is $\$ 18.00$ per article (or portion thereof), which should be paid directly to the Copyright Clearance Center (CCC), 222 Rosewood Drive, Danvers, MA 01923. Payment may also be made electronically through CCC Online at copyright.com. Other copying for republication, resale, advertising or promotion, or any form of systematic or multiple reproduction of any material in this book is prohibited except with permission in writing from the publisher. The CCC fee code is 0277-786X/10/\$18.00.

Printed in the United States of America.

Publication of record for individual papers is online in the SPIE Digital Library.

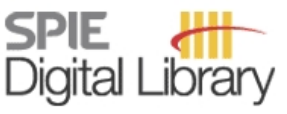

SPIEDigitalLibrary.org

Paper Numbering: Proceedings of SPIE follow an e-First publication model, with papers published first online and then in print and on CD-ROM. Papers are published as they are submitted and meet publication criteria. A unique, consistent, permanent citation identifier (CID) number is assigned to each article at the time of the first publication. Utilization of CIDs allows articles to be fully citable as soon they are published online, and connects the same identifier to all online, print, and electronic versions of the publication. SPIE uses a six-digit CID article numbering system in which:

- The first four digits correspond to the SPIE volume number.

- The last two digits indicate publication order within the volume using a Base 36 numbering system employing both numerals and letters. These two-number sets start with 00, 01, 02, 03, 04 , $05,06,07,08,09,0 A, 0 B \ldots$. OZ, followed by 10-1Z, 20-2Z, etc.

The CID number appears on each page of the manuscript. The complete citation is used on the first page, and an abbreviated version on subsequent pages. Numbers in the index correspond to the last two digits of the six-digit CID number. 


\section{Contents}

ix Conference Committee

xi Ultrafast fiber laser technology: status and prospects (Plenary Paper) [7579-102]

A. Tünnermann, J. Limpert, Friedrich-Schiller-Univ. Jena (Germany) and Fraunhofer-Institute for Applied Optics and Precision Engineering (Germany)

\section{SESSION 1 LASER DIODE RELIABILITY}

758302 Reliability of high performance 9xx-nm single emitter laser diodes [7583-01]

L. Bao, J. Wang, M. DeVito, D. XU, D. Wise, P. Leisher, M. Grimshaw, W. Dong, S. Zhang,

K. Price, D. Li, C. Bai, S. Patterson, R. Martinsen, nLIGHT Corp. (United States)

758303 Highly reliable 637-639-nm red high-power LDs for displays [7583-02]

T. Nishida, N. Shimada, K. Ono, T. Yagi, A. Shima, Mitsubishi Electric Corp. (Japan)

758304 Reliability of high-power QCW arrays [7583-03]

R. Feeler, J. Junghans, J. Remley, D. Schnurbusch, E. Stephens, Northrop Grumman Cutting Edge Optronics (United States)

758305 Reliability and performance of 808-nm single emitter multi-mode laser diodes [7583-04]

J. Wang, L. Bao, M. DeVito, D. Xu, D. Wise, M. Grimshaw, W. Dong, S. Zhang, C. Bai, P. Leisher,

D. Li, H. Zhou, S. Patterson, R. Martinsen, J. Haden, nLIGHT Corp. (United States)

758306 Reliable operation of 8xx mini-bar-based hermetic modules [7583-05]

X. Jin, S. Cutillas, D. Liu, E. Wolak, S.-K. Park, K. Johnson, T. Towe, D. Lenarduzzi, T. Nguyen,

T. Truchan, J. Mott, J. Harrison, Oclaro, Inc. (United States); A. Guarino, J. Müller, S. Pawlik,

B. Sverdlov, N. Lichtenstein, Oclaro Switzerland AG (Switzerland); C. Button, Oclaro

Technology plc (United Kingdom)

758307 Root cause investigation of catastrophic degradation in high power multi-mode InGaAs-AIGaAs strained quantum well lasers [7583-06]

Y. Sin, N. Ives, N. Presser, S. C. Moss, The Aerospace Corp. (United States)

\section{SESSION 2 LASER DIODE MODULES I}

$758308 \quad$ High brightness fiber-coupled pump laser development [7583-07]

K. Price, S. Karlsen, P. Leisher, R. Martinsen, nLIGHT Corp. (United States)

758309 Roadmap to low cost high brightness diode laser power out of the fiber [7583-08] D. Schröder, E. Werner, A. Franke, L. Wagner, G. Bonati, JENOPTIK Laserdiode GmbH (Germany); F. Dörfel, H. Ziemer, A. Liem, T. Gabler, JT Optical Engine GmbH + Co. KG (Germany) 
7583 OA High-brightness 9XX-nm pumps with wavelength stabilization [7583-09]

V. Gapontsev, N. Moshegov, P. Trubenko, A. Komissarov, I. Berishev, O. Raisky, N. Strougov,

V. Chuyanov, O. Maksimov, A. Ovtchinnikov, IPG Photonics Corp. (United States)

7583 OB Dramatic advances in direct diode lasers [7583-10]

D. Havrilla, TRUMPF Inc. (United States); M. Holzer, R. Brockmann, S. Strohmaier, TRUMPF Laser GmbH \& Co. KG (Germany)

\section{SESSION 3 LASER DIODE MODULES II}

7583 OC High duty cycle hard soldered kilowatt laser diode arrays [7583-1 1]

G. Klumel, Y. Karni, J. Oppenheim, Y. Berk, M. Shamay, R. Tessler, S. Cohen, SemiConductor Devices (Israel)

7583 OD Micro-optic solutions for beam shaping of individually addressable high-power single-mode diode laser arrays [7583-12]

M. Forrer, D. Kura, E. Langenbach, FISBA OPTIK AG (Switzerland)

7583 OE KW-class industrial diode lasers comprised of single emitters [7583-13]

K. Price, F. Pfeffer, P. Leisher, S. Karlsen, R. Martinsen, nLIGHT Corp. (United States)

7583 OF High-power diode laser modules from $410 \mathrm{~nm}$ to $2200 \mathrm{~nm}$ [7583-14]

B. Köhler, H. Kissel, M. Flament, P. Wolf, T. Brand, J. Biesenbach, DILAS Diodenlaser GmbH (Germany)

7583 OG Fiber coupling of high-power diode laser stack for direct polycarbonate processing [7583-15]

E. Vidal, I. Quintana, U. Azkorbebeitia, Fundacion Tekniker (Spain); E. Mendez, G. Viera,

M. Galán, MONOCROM S.L. (Spain); D. Otaduy, Fundacion Tekniker (Spain)

\section{SESSION 4 LASER DIODE HEAT REMOVAL}

$7583 \mathrm{OH} \quad$ Expansion matched heat sinks made by $\boldsymbol{\mu}$-metal injection molding [7583-16] M. Leers, E. Liermann, Fraunhofer Institute for Laser Technology (Germany); P. Imgrund, L. Kramer, J. Volkert, Fraunhofer Institute for Manufacturing \& Advanced Materials (Germany)

7583 ol Simple method for modeling thermoelectric cooler (TEC) performance of single-emitter semiconductor-laser packages with concentrated heat sources [7583-17]

J. Du, M. Au, L. Zavala, P. Yalamanchili, J. Skidmore, E. Zucker, JDSU (United States)

7583 0J Development of asymmetric epitaxial structures for $65 \%$ efficiency laser diodes in the 9xxnm range [7583-50]

M. Levy, Y. Karni, N. Rapaport, Y. Don, Y. Berk, D. Yanson, S. Cohen, J. Oppenheim, SemiConductor Devices (Israel)

7583 OK Progress in the development of active heat sink for high-power laser diodes [7583-19] J. Vetrovec, Aqwest LLC (United States); R. Feeler, S. Bonham, Northrop-Grumman Cutting Edge Optronics (United States) 
$7583 \mathrm{OL}$ Recent development of high-power-efficiency 50-W CW TE/TM polarized 808-nm diode laser bar at Lasertel [7583-20]

C. Cao, L. Fan, I. Ai, J. Li, B. Caliva, L. Zeng, P. Thiagarajan, M. McElhinney, Lasertel, Inc. (United States)

$75830 \mathrm{M}$ Extending the wavelength range in the Oclaro high-brightness broad area modules [7583-21]

S. Pawlik, A. Guarino, B. Sverdlov, J. Müller, Oclaro Switzerland AG (Switzerland); C. Button, Oclaro Technology, plc (United Kingdom); S. Arlt, D. Jaeggi, N. Lichtenstein, Oclaro,

Switzerland AG (Switzerland)

$7583 \mathrm{ON} \quad$ 975-nm high-power broad area diode lasers optimized for narrow spectral linewidth applications [7583-22]

P. Crump, C. M. Schultz, A. Pietrzak, S. Knigge, O. Brox, A. Maaßdorf, F. Bugge, H. Wenzel,

G. Erbert, Ferdinand-Braun-Institut für Höchstfrequenztechnik (Germany)

$758300 \quad$ Mid-infrared high-power diode lasers and modules [7583-23]

M. T. Kelemen, J. Gilly, m2k-laser GmbH (Germany); M. Rattunde, J. Wagner,

Fraunhofer-Institut für Angewandte Festkörperphysik (Germany); S. Ahlert, J. Biesenbach,

DILAS Diodenlaser GmbH (Germany)

$7583 \mathrm{OP} \quad$ High temperature and high peak-power 808-nm QCW bars and stacks [7583-24]

G. Bacchin, Intense Ltd. (United Kingdom); A. Fily, Intense-US (United States); B. Qiu, D. Fraser,

S. Robertson, Intense Ltd. (United Kingdom); V. Loyo-Maldonado, Intense-US (United States);

S. D. McDougall, B. Schmidt, Intense Ltd. (United Kingdom)

$7583 \mathrm{OQ}$ Eye safe high power laser diode in the 1410-1550-nm range [7583-25]

J. Boucart, Oclaro Switzerland AG (Switzerland); B. de Largy, M. Kearley, Oclaro Technology plc (United Kingdom); N. Lichtenstein, Oclaro Switzerland AG (Switzerland)

\section{SESSION 6 LASER DIODE DEVICES II}

7583 OR High power DFB laser diodes [7583-26]

W. Zeller, nanoplus GmbH (Germany); M. Kamp, Julius-Maximilians-Univ. Würzburg

(Germany); J. Koeth, nanoplus GmbH (Germany)

7583 OS Comparison of concepts for high-brightness diode lasers at $976 \mathrm{~nm}$ [7583-27]

J. Gilly, P. Friedmann, m2k-laser GmbH (Germany); H. Kissel, J. Biesenbach, DILAS

Diodenlaser GmbH (Germany); M. T. Kelemen, m2k-laser GmbH (Germany)

7583 OT Scaling brilliance of high power laser diodes [7583-28]

H. König, G. Grönninger, C. Laver, W. Reill, M. Arzberger, U. StrauB, OSRAM Opto Semiconductors GmbH (Germany); H. Kissel, J. Biesenbach, DILAS Diodenlaser GmbH (Germany); A. Kösters, J. Malchus, V. Krause, Laserline GmbH (Germany)

7583 OU JENOPTIK diode lasers and bars optimized for high-power applications in the NIR range [7583-29]

M. Zorn, R. Hülsewede, H. Schulze, J. Sebastian, JENOPTIK Diode Lab GmbH (Germany);

P. Hennig, D. Schröder, JENOPTIK Laserdiode GmbH (Germany) 
7583 OV High-brightness 635-nm tapered diode lasers with optimized index guiding [7583-30]

D. Feise, G. Blume, H. Dittrich, C. Kaspari, K. Paschke, G. Erbert, Ferdinand-Braun-Institut für Höchstfrequenztechnik (Germany)

7583 OW Characteristics of red-emitting broad area stripe laser diodes with zinc diffused window structures [7583-31]

T. Ohno, M. Takiguchi, K. Wakabayashi, H. Uchida, K. Naganuma, M. Ohara, S. Ito, S. Hirata, Sony Corp. (Japan)

\section{SESSION 8 BEAM COMBINING}

7583 OY Demonstration of high pointing accuracy dual-axis collimation of $\mathbf{4 9}$ emitter diode bar using a laser-written custom phase-plate [7583-33]

N. Trela, H. J. Baker, Heriot-Watt Univ. (United Kingdom); J. J. Wendland, PowerPhotonic, Ltd. (United Kingdom); D. R. Hall, Heriot-Watt Univ. (United Kingdom)

$75830 Z$ Coherent beam combining of high-power broad-area laser diode array in CW and pulsed modes [7583-34]

B. Liu, Y. Liu, Y. Braiman, Oak Ridge National Lab. (United States)

758310 Progress in high-brightness diode laser development based on tailored diode laser bars [7583-35]

S. D. Roh, D. M. Grasso, N. Shou, R. Pathak, G. Cheung, D. Schleuning, T. Hasenberg, Coherent, Inc. (United States)

758311 Coherent polarization locking of a diode emitter array [7583-36]

S. P. Ng, Nanyang Technological Univ. (Singapore); P. B. Phua, Nanyang Technological Univ. (Singapore) and DSO National Labs. (Singapore)

758312 High-brightness emission from stripe-array broad area diode lasers operated in off-axis external cavities [7583-37]

A. Jechow, D. Skoczowsky, Univ. of Potsdam (Germany); M. Lichtner, M. Radziunas, Weierstrass Institute for Applied Analysis and Stochastics (Germany); R. Menzel, Univ. of Potsdam (Germany)

$758313 \quad 250 W$ diode laser for low pressure Rb vapor pumping [7583-38]

A. Podvyaznyy, G. Venus, CREOL, The College of Optics and Photonics, Univ. of Central Florida (United States); V. Smirnov, O. Mokhun, V. Koulechov, OptiGrate Corp. (United States); D. Hostutler, Air Force Research Lab. (United States); L. Glebov, CREOL, The College of Optics and Photonics, Univ. of Central Florida (United States) 
758314 Degradation analysis of individual emitters in 808-nm QCW laser diode array for space applications [7583-40]

O. Rehioui, IMS-Labs., CNRS, Univ. Bordeaux (France) and Quantel Laser Diodes (France); L. Bechou, IMS-Labs., CNRS, Univ. Bordeaux (France); T. Fillardet, A. Kohl, Quantel Laser Diodes (France); Y. Ousten, IMS-Labs., CNRS, Univ. Bordeaux (France); G. Volluet, Quantel Laser Diodes (France)

758317 High-power distributed-feedback tapered master-oscillator power amplifiers emitting at $1064 \mathrm{~nm}$ [7583-43]

D. Jedrzejczyk, O. Brox, F. Bugge, J. Fricke, A. Ginolas, K. Paschke, H. Wenzel, G. Erbert, Ferdinand-Braun-Institut für Höchstfrequenztechnik (Germany)

$758318 \quad$ CW to QCW power scaling of high-power laser bars [7583-44]

J. Müller, R. Todt, M. Krejci, Y. Manz-Gilbert, B. Valk, R. Brunner, R. Bättig, N. Lichtenstein, Oclaro Switzerland AG (Switzerland)

758319 1043-nm semiconductor disk laser [7583-45]

Y. Song, P. Zhang, J. Tian, X. Zhang, Beijing Univ. of Technology (China)

75831 1A High-power high-brightness 808-nm QCW laser diode mini bars [7583-46]

H. Huang, J. Wang, M. DeVito, L. Bao, A. Hodges, S. Zhang, D. Wise, M. Grimshaw, D. Xu, C. Bai, nLIGHT Corp. (United States)

$75831 \mathrm{E}$ Laser-assisted shearing: new application for high-power diode lasers [7583-53] M. Emonts, C. Brecher, Fraunhofer-Institut für Produktionstechnologie (Germany)

Author Index 
Downloaded From: https://www.spiedigitallibrary.org/conference-proceedings-of-spie on 26 Apr 2023

Terms of Use: https://www.spiedigitallibrary.org/terms-of-use 


\title{
Conference Committee
}

\author{
Symposium Chairs
}

Donald J. Harter, IMRA America, Inc. (United States)

Peter R. Herman, University of Toronto (Canada)

Symposium Cochairs

Alberto Piqué, Naval Research Laboratory (United States)

Friedhelm Dorsch, TRUMPF Photonics (United States)

Program Track Chair

Klaus P. Streubel, OSRAM Opto Semiconductors GmbH (Germany)

Conference Chair

Mark S. Zediker, Consultant (United States)

Program Committee

Friedrich G. Bachmann, Rofin-Sinar Laser GmbH (Germany)

Stefan W. Heinemann, Fraunhofer USA, Inc. (United States)

Volker K. Krause, Laserline GmbH (Germany)

Robert J. Martinsen, nLIGHT Corporation (United States)

Kurt J. Linden, Spire Corporation (United States)

Erik P. Zucker, JDSU (United States)

\section{Session Chairs}

1 Laser Diode Reliability

Robert J. Martinsen, nLIGHT Corporation (United States)

2 Laser Diode Modules I

Erik P. Zucker, JDSU (United States)

3 Laser Diode Modules II

Friedrich G. Bachmann, Rofin-Sinar Laser GmbH (Germany)

$4 \quad$ Laser Diode Heat Removal

Stefan W. Heinemann, Fraunhofer USA, Inc. (United States) 
$5 \quad$ Laser Diode Devices I

Erik P. Zucker, JDSU (United States)

6 Laser Diode Devices II

Kurt J. Linden, Spire Corporation (United States)

7 Laser Diode Devices III

Kurt J. Linden, Spire Corporation (United States)

$8 \quad$ Beam Combining

Volker K. Krause, Laserline GmbH (Germany) 